\title{
Glycemic variability and glucose complexity in critically ill patients: a retrospective analysis of continuous glucose monitoring data
}

\author{
Richard Brunner ${ }^{1}$, Gabriel Adelsmayr ${ }^{1}$, Harald Herkner ${ }^{2}$, Christian Madl ${ }^{1}$ and Ulrike Holzinger ${ }^{1 *}$
}

\begin{abstract}
Introduction: Glycemic variability as a marker of endogenous and exogenous factors, and glucose complexity as a marker of endogenous glucose regulation are independent predictors of mortality in critically ill patients. We evaluated the impact of real time continuous glucose monitoring (CGM) on glycemic variability in critically ill patients on intensive insulin therapy (IIT), and investigated glucose complexity - calculated using detrended fluctuation analysis (DFA) - in ICU survivors and non-survivors.

Methods: Retrospective analysis were conducted of two prospective, randomized, controlled trials in which 174 critically ill patients either received IIT according to a real-time CGM system $(n=63)$ or according to an algorithm $(n=111)$ guided by selective arterial blood glucose measurements with simultaneously blinded CGM for 72 hours. Standard deviation, glucose lability index and mean daily delta glucose as markers of glycemic variability, as well as glucose complexity and mean glucose were calculated.
\end{abstract}

Results: Glycemic variability measures were comparable between the real time CGM group $(n=63)$ and the controls $(n=111)$. Glucose complexity was significantly lower (higher DFA) in ICU non-survivors $(n=36)$ compared to survivors $(n=138)$ (DFA: 1.61 (1.46 to 1.68$)$ versus $1.52(1.44$ to 1.58$) ; P=0.003)$. Diabetes mellitus was significantly associated with a loss of complexity (diabetic $(n=33)$ versus non-diabetic patients $(n=141)$ (DFA: 1.58 (1.48 to 1.65 ) versus 1.53 (1.44 to 1.59$) ; P=0.01$ ).

Conclusions: IIT guided by real time CGM did not result in significantly reduced glycemic variability. Loss of glucose complexity was significantly associated with mortality and with the presence of diabetes mellitus.

\section{Introduction}

Glucose control in critically ill patients has been a highly disputed topic since 2001, when van den Berghe et al. showed that intensive insulin therapy (IIT) (mean glucose levels $\leq 6.11 \mathrm{mmol} / \mathrm{L}$ ) could reduce the morbidity and mortality of patients in surgical ICUs by $42 \%$ [1]. However, subsequent studies came to inconclusive findings [2]. Recently, several retrospective trials found glycemic variability per se to be associated with mortality in critically ill patients, independent of mean glucose concentration [3-9].

The measure glycemic variability describes fluctuations of blood glucose over time. As glucose fluctuations are not

\footnotetext{
* Correspondence: ulrike.holzinger@meduniwien.ac.at

'Department of Medicine III, Division of Gastroenterology and Hepatology, ICU 13H1, Medical University of Vienna, Waehringer Guertel 18-20, 1090 Vienna, Austria

Full list of author information is available at the end of the article
}

covered by mean glucose, glycemic variability has been suggested as an additional measure for glucose control. Glycemic variability is represented by standard deviation (SD), mean daily $\delta$ blood glucose or glucose lability index (GLI). SD is the most commonly used parameter and is calculated as the square-root of the average of the squared differences between individual glucose values and the mean. Mean daily $\delta$ blood glucose describes the mean of the daily difference between minimum and maximum blood glucose. These two measures do not take order and timing of measurements into account. GLI is the squared difference between consecutive blood glucose levels per unit of actual time between the samples. GLI considers the time between and the order of measurements. Although no gold standard of measuring glycemic variability has been established yet, SD seems to be the best predictor of mortality [10]. 
In a prospective study of septic critically ill patients a significant association between high glycemic variability and mortality was found [11]. These results are consistent with in vitro data showing that short-time fluctuations of glucose levels induce endothelial cell damage and apoptosis [12]. Moreover, a significant association between glycemic variability and 8-iso prostaglandin $F_{2 \alpha}$, a marker of oxidative stress and potential mediator of organ dysfunction, has been shown in diabetic type 2 patients [13]. Minimal glycemic variability has been proposed to become the gold standard of glycemic control in diabetic patients [14].

Glycemic variability depends on both endogenous patient-specific factors such as severity of disease and diabetes status [15], as well as exogenous factors such as type and quality of glucose monitoring, the glucose algorithm used for the calculation of the insulin rate, compliance of the nursing staff with the recommendations of the protocol and application of medication including enteral and parenteral nutrition. As the endogenous glucose regulation system can hardly be influenced, glycemic variability needs to be improved by acting on the exogenous factors. Besides well-trained nursing staff and continuous application of medication, appropriate glucose monitoring is suggested to minimize blood glycemic variability. Therefore, the need for real time continuous glucose level reporting has been emphasized numerous times $[16,17]$. Subcutaneous continuous glucose monitoring (CGM) systems provide both real time capability and adequate accuracy in medical critically ill patients including those requiring vasopressors [18].

Although IIT may be associated with increased glycemic variability [7] we hypothesized that IIT guided by real time glucose monitoring would decrease glycemic variability. Thus, we aimed to evaluate the impact of real time CGM on glycemic variability in critically ill patients.

More importantly, by using CGM devices valuable insights into glucose regulation are possible. While glucose variability can be calculated using conventional blood glucose measurements every four to six hours, glucose complexity calculation requires the availability of continuous glucose data. Recently, in critically ill patients glucose complexity has been proposed as a marker of endogenous glucose regulation [19].

Glucose complexity is a dynamic measure of glucose time series and, therefore, seems to provide more powerful information on endogenous glucose regulation than does conventional glycemic variability analysis. In contrast to glycemic variability that describes the magnitude of glucose fluctuations over several hours, glucose complexity is proposed as a measure of short-term glucose oscillations. Glycemic variability depends on endogenous and exogenous factors, whereas glucose complexity is proposed as a description of the endogenous glucose regulation system that is independent from exogenous factors. Lundelin et al. hypothesized that in a healthy regulatory system, glucose levels are corrected frequently and result in a 'complex' glucose profile [19]. However, in critically ill patients a decomplexification of glucose regulation was suggested [20]. Low complexity represents the inability of the patient to correct glucose fluctuations frequently and quickly.

In a small number of patients glycemic profile was shown to be more complex in ICU survivors than in ICU non-survivors [19]. However, this has not been confirmed in a large group of critically ill patients. Therefore, we investigated the role of glucose complexity in a large group of critically ill patients.

The primary hypothesis is that real-time CGM guidance of IIT is associated with decreased glycemic variability in critically ill patients. The secondary hypothesis of the study is that glucose complexity is independently associated with increased mortality.

\section{Materials and methods}

Data analysis was approved by the ethics committee of the Medical University of Vienna. Because of the retrospective character of the analysis the need for informed consent was waived by the institutional review board.

\section{Patients and setting}

This is a post-hoc analysis of two prospective, randomized controlled trials conducted in an eight-bed closed medical ICU at the University Hospital of Vienna, Austria $[21,22]$. During the period from April 2005 to August 2008 a total of 983 critically ill patients were admitted, 728 of whom were ventilated. A total of 174 consecutive, mechanically ventilated and sedated patients fulfilling the inclusion criteria (age $\geq 18$, expected to stay $\geq 48$ hours in the ICU after initiation of IIT) were enrolled in the study within 48 hours after ICU admission. Patients were not enrolled in the study if any of the following criteria were present: ICU stay expected to be $<48$ hours, mechanical ventilation not expected for $>48$ hours, inclusion in another study, no CGM device available during the screening phase or glucose values in the normal range without insulin therapy.

The original objectives of the two studies were to evaluate the impact of circulatory shock requiring norepinephrine therapy on the accuracy and reliability of a subcutaneous CGM sensor in critically ill patients [21] and to evaluate the impact of real-time CGM on glycemic control and risk of hypoglycemia in critically ill patients [22].

\section{Research design}

To evaluate the impact of real time CGM on glucose variability, data on patients allocated to two groups (real 
time CGM, $\mathrm{n}=63$ and controls, $\mathrm{n}=111$ ) were analyzed.

All included patients were treated with IIT to maintain glucose levels between 4.44 and $6.11 \mathrm{mmol} / \mathrm{L}$.

In the control group insulin infusion rates were guided by selective arterial blood glucose measurements, obtained using an automated blood gas analyzer (Radiometer ABL $700^{\circledR}$, Copenhagen, Denmark). IIT was performed by the nursing staff according to a previously described dynamic paper-based insulin titration algorithm [23] based on the algorithm used in the Leuven studies $[1,24]$. This algorithm prescribes the insulin infusion rate, time of next glucose measurement (between one and six hours), and, in the case of hypoglycemia, dextrose administration depending on glucose levels and glucose trends. Consequently, it defines nine different states requiring different actions, although leaving space for interpretation by the responsible nurse [22]. In the control group glucose levels were additionally recorded continuously using the Continuous Glucose Monitoring System $^{\circledR}$ (CGMS, Medtronic MiniMed, Northridge, CA, USA), but were blinded and available only in retrospect.

In the real time CGM group, IIT was performed by the nursing staff and insulin infusion rates were guided by continuously available glucose levels using the Guardian ${ }^{\circledR}$ real time CGMS (Medtronic MiniMed) according to the algorithm used in the control group. In contrast to the control group, nurses were requested to take real time glucose readings in close intervals according to clinical necessity at personal discretion, however at least every two hours [22].

To investigate glucose complexity detrended fluctuation analysis (DFA) [19] was calculated for ICU survivors $(\mathrm{n}=138)$ and ICU non-survivors $(\mathrm{n}=36)$. Furthermore, we evaluated glucose complexity in diabetic $(\mathrm{n}=33)$ and non-diabetic $(\mathrm{n}=141)$ patients.

\section{Real time continuous glucose monitoring system (real time CGMS)}

The Guardian ${ }^{\circledR}$ real time CGMS has been described in detail previously [22]. Briefly, it displays a mean of 30 glucose measurements over the last five minutes on a monitor, allowing glucose monitoring in real time. The real time CGMS was calibrated against blood glucose measurements, obtained using an automated blood gas analyzer, at least four times per day (every five to six hours). Sensors were planned to stay in place for 72 hours.

\section{Continuous glucose monitoring system (CGMS)}

The CGMS has been described in detail previously [22]. Briefly, it is equivalent to the Guardian ${ }^{\circledR}$ real time CGMS, except it is lacking the capability to display glucose concentration in real time. Glucose concentrations were recorded continuously in an internal monitor blinded to the study team and obtained after the trial for further analysis.

\section{Statistical analysis}

To evaluate the impact of real time CGM on glucose variability as a marker of exogenous glucose regulation, we used linear regression analyses with glycemic variability, represented as SD (Glu $\left.u_{\mathrm{SD}}\right)$, glucose lability index $\left(\left\{\sum\left(\mathrm{Glu}_{\mathrm{n}}-\mathrm{Glu}_{\mathrm{n}+1}(\mathrm{mmol} / \mathrm{L})\right)^{2 * *}\left(\mathrm{~h}_{\mathrm{n}+1}-\mathrm{h}_{\mathrm{n}}\right)^{-1}\right)^{*}(\right.$ number of readings) ${ }^{-1}$ or mean daily delta (difference between minimum and maximum) glucose as primary outcome and real time versus concealed CGMS (controls) as predictor.

Secondary endpoints were coefficient of variation $(\mathrm{CV})$ of glucose $\left(\mathrm{Glu}_{\mathrm{SD}} / \mathrm{Glucose}\right.$ mean (\%)), variability or mean of glucose during the first 24 hours, maximum glucose during ICU stay.

To investigate glycemic dynamics and its relation with mortality in critically ill patients we used glucose complexity as the main risk factor and ICU mortality as outcome. Glucose complexity is proposed as the representation of the endogenous glucoregulatory process. Although it is similar to glucose variability it is able to detect minor systemic alterations in endogenous glucose regulation. In a healthy regulatory system, glucose levels are corrected frequently and result in a 'complex' glucose profile. However, in critically ill patients a decomplexification of glucose regulation was suggested [20].

Glucose complexity was calculated using DFA. DFA is a unitless metric that estimates the degree of long-range correlations within a signal, analyzing how the time series and its linear regression diverge as the 'time window' considered increases. As a rule of thumb, higher complexities are displayed as lower DFA (until a minimum of 0.5). Details on DFA can be found elsewhere [19].

Glucose complexity was normally distributed as assessed by visual inspection of a histogram but not linearly related with mortality as assessed with a test for deviation from linearity in a logistic regression model. We found a good fit with a quadratic function and entered a quadratic term into the model as a consequence. We assessed whether this association was modified by diabetes by testing the significance of the interaction term in such a model using a Wald test. To allow for the influence of other variables on the effect of glucose complexity we entered SAPS II (Simplified Acute Physiology Score) score, age (years), sex, and diabetes (yes versus no) as covariables into our model. These covariables were selected $a$ priori. As we merged patients from two studies into this database we used a multivariate mixed effects logistic regression model to additionally allow for potential clustering within each of the two studies. As sensitivity analyses we used robust estimations instead which yielded virtually the same results. 
We assessed the impact of the method of glucose determination (CGMS versus blood gas analyzer (BGA)) on the glucose variability measures SD, CV, GLI and mean daily delta glucose by calculating them from CGMS and BGA values in all patients.

Data are presented as mean \pm standard deviation, median $\left(25^{\text {th }}\right.$ to $75^{\text {th }}$ percentile) or absolute count and relative frequency. For bivariate comparisons we tabulated data and used simple one-way analysis of variance (ANOVA), Mann-Whitney rank sum test or a chi2test as appropriate to test the null hypothesis of no difference.

For data management and analyses we used Excel for Mac 2011 and STATA 11.0 for Mac (Stata Corp., College Station, TX, USA). Generally a two-sided $P$-value $<0.05$ was considered statistically significant.

\section{Results}

\section{Baseline characteristics}

Baseline characteristics of 174 critically ill patients receiving IIT either guided by a real time CGM system or by selective arterial blood glucose measurements with simultaneously blinded CGM can be found in Table 1. Mean CGM time was $59.2 \pm 14.7$ hours (RT CGM group); $7.0 \pm 1.6 \mathrm{BGA}$ readings per patient in 24 hours were taken in the control group.

\section{Differences in glycemic metrics between patients using} real time CGM and controls

The use of real time CGM did not have any impact on the measures of glycemic variability, glucose complexity and maximum glucose (Table 2).

\section{Differences in glycemic metrics between ICU survivors} and non-survivors

Measures of glycemic variability, mean glucose and hypoglycemia were similar between ICU survivors and ICU non-survivors, whereas glucose complexity was significantly lower in non-survivors (Table 3).

These measures were similar in hospital survivors and non-survivors (data not shown).

\section{Diabetic status and glucose complexity}

The presence of diabetes was significantly associated with a loss of complexity (higher DFA) (diabetic $(n=33)$ versus non-diabetic patients $(\mathrm{n}=141)$ : DFA $1.58(1.48$ to 1.65$)$ versus 1.53 (1.44 to 1.59 ); $P=0.01$ ). This difference persisted even after correcting for survival $(P=0.027)$.

Multivariate analysis of glucose complexity and mortality Although glucose complexity was significantly lower in non-survivors, relation between glucose complexity and mortality was not linear but can be described best with a quadratic function: logodds (ICU survival) $=(-0.09 *$ DFA decile $)^{2}+0.64 *$ DFA decile +1.07 , where DFA decile (from 0 to 9 ) represents one tenth of DFA values in increasing sequence (Figure 1).

The difference of glucose complexity between survivors and non-survivors was confirmed in a binary logistic regression analysis with ICU mortality as outcome and glucose complexity, as well as age, BMI, gender, diabetes status and SAPS II as co-factors. In this model only DFA was significantly associated with mortality.

The glycemic variability measures SD, CV and GLI were significantly higher while mean daily delta glucose

Table 1 Admission reason and baseline characteristics.

\begin{tabular}{lccc}
\hline & Real time CGM [21] & Controls [21,22] & Total [21,22] \\
\hline Included Patients & 63 & 111 & 174 \\
Admission reason & & Number of patients (\% of patients in the category) & $38(22)$ \\
Respiratory failure & $15(24)$ & $23(21)$ & $39(22)$ \\
CPR & $12(19)$ & $27(24)$ & $37(21)$ \\
Sepsis/Septic shock & $13(20)$ & $24(22)$ & $29(17)$ \\
Heart failure & $8(13)$ & $21(19)$ & $19(11)$ \\
Neurologic disease/Coma & $9(14)$ & $10(9)$ & $6(3)$ \\
Pulmonary embolism & $3(5)$ & $3(3)$ & $5(3)$ \\
Gl-bleeding/ALF & $3(5)$ & $2(2)$ & $1(1)$ \\
Necrotising pancreatitis & $0(0)$ & $21(19)$ & $33(19)$ \\
History of diabetes & $12(19)$ & $62 \pm 16$ & $61 \pm 16$ \\
Age (years) & $59 \pm 15$ & $64 / 47$ & $105 / 69$ \\
Gender (male/female) & $41 / 22$ & $26.4 \pm 3.9$ & $26.7 \pm 4.4$ \\
BMI (kg/m²) & $27.1 \pm 5.1$ & $58 \pm 16$ & $11 \pm 4$ \\
SAPS II & $60 \pm 16$ & $10.9 \pm 3.5$ & $59 \pm 16$ \\
SOFA on admission day & $11.5 \pm 3.8$ &
\end{tabular}

Data are means \pm SD, unless otherwise stated. ALF, acute liver failure; BMI, body mass index; CPR, Cardiopulmonary resuscitation; SAPSIl, Simplified Acute Physiology Score; SD, standard deviation; SOFA, Sequential Organ Failure Assessment. 
Table 2 Glycemic metrics in the real time CGM and control group.

\begin{tabular}{|c|c|c|c|}
\hline & $\begin{array}{c}\text { Real time CGM } \\
\text { (number = 63) }[21]\end{array}$ & $\begin{array}{c}\text { Controls } \\
\text { (number }=111)[21,22]\end{array}$ & $P$-value \\
\hline \multicolumn{4}{|l|}{ Measures of glycemic variability } \\
\hline Variability of glucose (SD) (mmol/L) & $1.19 \pm 0.49$ & $1.27 \pm 0.54$ & 0.330 \\
\hline Variability of glucose (GLI) & 81 (43 to 197) & 126 (64 to 222) & 0.247 \\
\hline Variability of glucose $(\delta)(\mathrm{mmol} / \mathrm{L})$ & $4.47 \pm 2.02$ & $4.76 \pm 2.07$ & 0.336 \\
\hline Coefficient of variation (\%) & $20 \pm 7$ & $21 \pm 8$ & 0.547 \\
\hline Variability of glucose during first 24 hours (SD) (mmol/L) & 0.84 (0.65 to 1.33$)$ & $1.04(0.66$ to 1.40$)$ & 0.395 \\
\hline Variability of glucose during first 24 hours (GLI) & 85 (38 to 190) & 118 (60 to 207) & 0.348 \\
\hline Variability of glucose during first 24 hours $(\delta)(\mathrm{mmol} / \mathrm{L})$ & $5.72 \pm 2.42$ & $5.73 \pm 2.40$ & 0.966 \\
\hline \multicolumn{4}{|l|}{ Measures of glucose } \\
\hline Mean of glucose during first 24 hours (mmol/L) & 5.70 (5.19 to 6.47$)$ & 5.96 (5.48 to 6.36$)$ & 0.099 \\
\hline Maximum glucose $(\mathrm{mmol} / \mathrm{L})$ & $9.43 \pm 2.12$ & $9.77 \pm 2.26$ & 0.329 \\
\hline Glucose complexity & $1.54 \pm 0.11$ & $1.52 \pm 0.11$ & 0.210 \\
\hline \multicolumn{4}{|l|}{ Measures of IIT } \\
\hline Cumulative daily dose of insulin (I.U.) & $45 \pm 27$ & $40 \pm 23$ & 0.239 \\
\hline Number of changes of the insulin infusion/24 hours & $3.8 \pm 1.5$ & $3.6 \pm 1.3$ & 0.573 \\
\hline Extent of insulin change $(\%)$ & $57 \pm 19$ & $62 \pm 23$ & 0.150 \\
\hline Number of BGA measurements/24 hours & $7.4 \pm 2.1$ & $7.0 \pm 1.6$ & 0.217 \\
\hline Number of BGA not required by the algorithm/24 $\mathrm{h}^{\mathrm{a}}$ & $0(0$ to 0$)$ & $0(0$ to 1$)$ & 0.238 \\
\hline
\end{tabular}

${ }^{\mathrm{a}}$ safety/double check BGA measurements. Data are shown as mean \pm SD or median $\left(25^{\text {th }}\right.$ to $75^{\text {th }}$ percentile). BGA, blood gas analyzer; CGM, continuous glucose monitoring; GLI, glucose lability index; IIT, intensive insulin therapy.

was significantly lower when calculated from BGA compared to CGMS values (Table 4).

\section{Discussion}

In this post-hoc analysis of CGM data the use of a real time CGM did not have an impact on measures of glycemic variability, glucose complexity and maximum glucose. The loss of glucose complexity was found to be independently associated with mortality and with the presence of diabetes mellitus.

\section{Glucose variability}

Glycemic variability describes fluctuations of blood glucose over time and is influenced by endogenous and

Table 3 Glycemic metrics in ICU survivors and non-survivors.

\begin{tabular}{|c|c|c|c|}
\hline & \multicolumn{2}{|c|}{ Real Time CGM and controls $[21,22]$} & \multirow[b]{2}{*}{$P$-value } \\
\hline & Survivors (number $=138$ ) & Non-survivors (number $=36$ ) & \\
\hline \multicolumn{4}{|l|}{ Measures of glycemic variability } \\
\hline Variability of glucose (SD) (mmol/L) & $1.21 \pm 0.49$ & $1.39 \pm 0.59$ & 0.067 \\
\hline Variability of glucose (GLI) & $112(62$ to 214$)$ & 126 (56 to 223$)$ & 0.468 \\
\hline Variability of glucose $(\delta)(\mathrm{mmol} / \mathrm{L})$ & $4.54 \pm 2.00$ & $5.08 \pm 2.20$ & 0.158 \\
\hline Coefficient of variation (\%) & $20 \pm 7$ & $22 \pm 8$ & 0.169 \\
\hline Variability of glucose during first 24 hours (SD) ( $\mathrm{mmol} / \mathrm{L})$ & 0.92 (0.64 to 1.32) & 1.03 (0.73 to 1.46$)$ & 0.083 \\
\hline Variability of glucose during first 24 hours (GLI) & 101 (54 to 200) & $123(54$ to 198$)$ & 0.232 \\
\hline Variability of glucose during first 24 hours $(\delta)(\mathrm{mmol} / \mathrm{L})$ & $5.55 \pm 2.23$ & $6.42 \pm 2.91$ & 0.051 \\
\hline \multicolumn{4}{|l|}{ Measures of glucose } \\
\hline Mean of glucose $(\mathrm{mmol} / \mathrm{L})$ & $6.03 \pm 0.57$ & $6.23 \pm 0.09$ & 0.097 \\
\hline Mean of glucose during first 24 hours (mmol/L) & 5.89 (5.40 to 6.36$)$ & 5.74 (5.27 to 6.39$)$ & 0.414 \\
\hline Maximum glucose (mmol/L) & $9.52 \pm 2.01$ & $10.16 \pm 2.82$ & 0.121 \\
\hline Glucose complexity & $1.51 \pm 0.10$ & $1.58 \pm 0.14$ & 0.003 \\
\hline \multicolumn{4}{|l|}{ Measures of hypoglycemia } \\
\hline Time below 4.44 mmol/L (min/24hours) & 109 (27 to 262$)$ & 120 (29 to240) & 0.874 \\
\hline Time below $3.33 \mathrm{mmol} / \mathrm{L}$ (min/24hours) & 0 (0 to 29) & 0 (0 to 50) & 0.864 \\
\hline Time below 2.22 mmol/L (min/24 hours) & $0(0$ to 0$)$ & $0(0$ to 0$)$ & 0.116 \\
\hline
\end{tabular}

Data are shown as mean \pm SD or median $\left(25^{\text {th }}\right.$ to $75^{\text {th }}$ percentile). CGM, continuous glucose monitoring; GLI, glucose lability index; SD, standard deviation. 


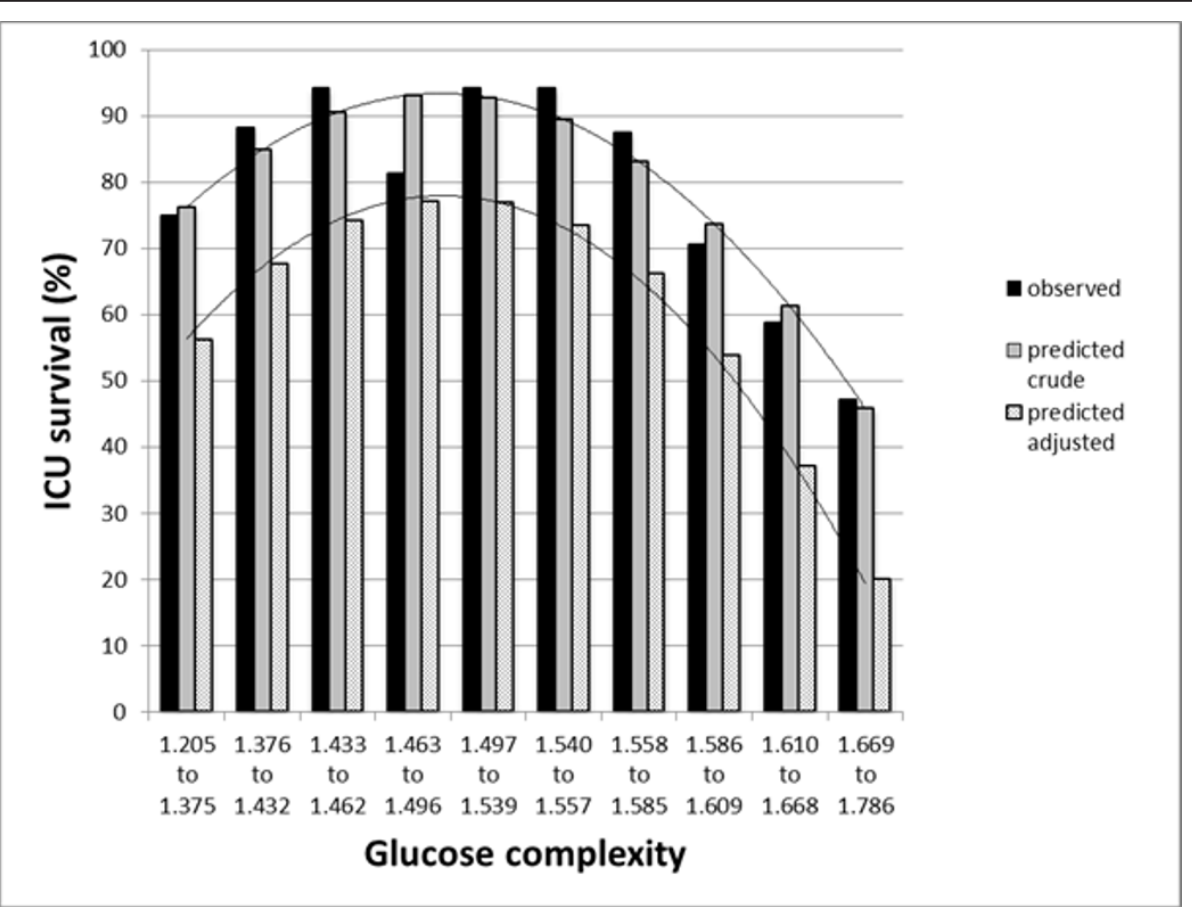

Figure 1 Relation between glucose complexity and mortality. Relation between glucose complexity and mortality can be described best with a quadratic function showing a pronounced increase in mortality with higher DFA and a moderate increase in mortality with very low DFA.

exogenous factors. It is associated with mortality and strategies are being sought for reducing glycemic variability. Improved glucose variability with real time CGM was reported in diabetic patients [25]. A possible explanation of real time CGM not reducing glycemic variability in the present study may be the use of an already well-established insulin titration algorithm in the control group. This algorithm has, in combination with the experienced nurses and frequent BGA measurements, already shown excellent results regarding glucose control. Hence, the use of real time CGM may have a larger benefit in environments with less experienced and established ICU staff.

Unlike numerous reports in the literature [3,4,8-10,26], we did not find a significant association between mortality and glycemic variability or between hypoglycemia and mortality, because our analysis was not powered for these purposes.

The method and frequency of glucose determination has a significant impact on variability measures as already shown with mean absolute glucose change per hour [27]. Mean daily delta was naturally higher when calculated from CGMS compared to BGA values as the gap between minimum and maximum glucose increases with the number of measured values. The increase in $\mathrm{SD}, \mathrm{CV}$ and GLI calculated from BGA values may be based on the fact that blood gases are taken more frequently when a patient's glucose levels are unstable, resulting in virtually higher glucose variability values. However, glucose variability measures were calculated from CGM values in both groups in our study. Therefore, measures between these groups are comparable.

\section{Glucose complexity}

Glucose complexity has been hypothesized as descriptive of the endogenous glucoregulatory process and is an independent predictor of mortality in critically ill patients as reported by Lundelin et al. for the first time [19]. These findings have now been confirmed in a larger

Table 4 Impact of the method of glucose determination on variability measures.

\begin{tabular}{lcc}
\hline Variability measures derived from: & $\begin{array}{c}\text { CGMS values } \\
\mathbf{( 1 7 4} \text { patients) }\end{array}$ & $\begin{array}{c}\text { BGA values value } \\
\text { (174 patients) }\end{array}$ \\
\hline Number of glucose measurements & 140209 & 3497 \\
GLI & $178 \pm 188$ & $301 \pm 380$ \\
Mean daily delta (mmol/L) & $4.65 \pm 2.06$ & $3.10 \pm 1.50$ \\
SD (mmol/L) & $1.24 \pm 0.52$ & $1.35 \pm 0.57$ \\
\hline
\end{tabular}

BGA, blood gas analyzer; CV, coefficient of variation; GLI, glucose lability index: SD, standard deviation. 
patient group in a medical ICU. Loss of complexity in glucose time series was significantly associated with higher mortality. However, the relation between glucose complexity and mortality was not linear but can be described best with a quadratic function with a pronounced increase in mortality with higher DFA and a moderate increase in mortality with very low DFA.

The underlying hypothesis is that the ability of a healthy organism to detect even minor changes in glucose concentration and then to follow promptly with counter regulatory measures leads to a complex glucose profile. In contrast, an impaired regulatory system responds slowly and imprecisely to varying glucose concentrations and, therefore, displays low glucose complexity [19]. The unexpected mortality increase with very complex profiles, which has not been discussed by Lundelin et al. [19], cannot be explained by the present data and needs further investigation. Therefore, the biological explanation of the association between glucose complexity and mortality in critically ill patients should still be seen as a hypothesis.

Glucose complexity, but not SAPS II score, was significantly associated with mortality in a binary logistic regression analysis. However, this study was not powered to address this association.

Moreover, the complexity of the glycemic profile was significantly lower in diabetic, compared to non-diabetic, critically ill patients. This is consistent with several studies assessing glucose complexity in non-critically ill diabetic patients $[28,29]$ and in critically ill patients after controlling for mortality [19]. Glucose complexity was similar between the real time CGM and the control group. We expected this finding, as glucose complexity reflects the endogenous fundamental glucose regulation, which seems autonomous from exogenous stimuli such as insulin treatment.

\section{Strengths and weaknesses}

The present findings may be influenced by the accuracy and method of glucose monitoring. Glucose variability and inaccuracy of glucose monitoring may be positively correlated [30] and glycemic variability may be underestimated with a higher time span between glucose measurements [31].

We regard the estimation of glycemic variability in our study acceptable based on the following factors: the CGMS is relatively accurate, the accuracy of the CGMS is constant at all glucose levels [18] and the time span between glucose measurements is very small (5 minutes).

Calculation of glucose complexity is possible only with CGM. The CGMS we used in the trial was the most accurate system available at that time.

Furthermore, glucose variability and complexity measures are calculated from CGMS values in all patients.
Thus, a systematic error based on the CGMS would influence both groups in the same way.

In our opinion, the advantages of the real time CGM could not be fully utilized based on the following factors. In our algorithm decisions are primarily based on the value of blood glucose, but not on the actual glucose trend [21]. However, glucose trend data is, in our opinion, one of the essential strengths of CGM. Therefore, we hypothesize that a (computer-based) algorithm using trend data for its decision process and capable of processing the great number of glucose values of CGM would be superior to the conventional algorithm used in our trials.

Moreover, the CGM devices in the study were used offlabel and were originally designed for outpatients. Therefore, the display was very small and trend data could not be visualized. Due to the study design and because of the impossibility of making alarms adequately audible in an ICU environment alarm functions were not used. Devices compensating for these shortcomings are currently under development by several manufacturers, but were not available when we conducted our trial.

Consequently, despite the availability of real time data, the frequency of changes of the insulin infusion did not increase in the CGM group (Table 2). Furthermore, the number of BGA measurements was equal in both groups. However, BGA are not only used for glucose measurement in our ICU. Based on these data we conclude that the use of our CGMS device did not have a significant impact on the behavior of the nursing staff in the real time CGM group compared to the control group.

Unlike in the study of Lundelin et al. [19], glucose complexity metrics were convincing in our analysis based on a relatively large patient group, standardized beginning of CGM and calibration of CGM devices with glucose values determined by very accurate blood gas analyzers [32].

\section{Conclusions}

IIT guided by real time CGM did not result in significantly reduced glycemic variability. The loss of glucose complexity was significantly associated with mortality and with the presence of diabetes mellitus. Thus, glucose complexity is an excellent measure of the endogenous glucose regulation and a robust parameter of the severity of disease in critically ill patients. In the future when continuous glucose monitoring may become standard in the ICU - glucose complexity may add to clinical scores in this regard.

\section{Key messages}

- IIT guided by real time CGM did not lead to reduced glycemic variability 
- The loss of glucose complexity is associated with mortality and diabetes mellitus in critically ill patients

\author{
Abbreviations \\ BGA: blood gas analyzer; CGM: continuous glucose monitoring; CGMS: \\ continuous glucose monitoring system; CV: coefficient of variation; DFA: \\ detrended fluctuation analysis; GLI: glucose lability index; Glu \\ glucose variability measured by SD; IIT: Intensive insulin therapy.
}

\section{Acknowledgements}

We thank all the health professionals of the $\mathrm{ICU} 13 \mathrm{H} 1$ for their commitment to this study, as well as Dr. Manuel Varela for providing us with the program to calculate glucose complexity.

This research did not receive any specific grant from any funding agency in the public, commercial or not-for-profit sector. Study equipment (two CGMS Gold monitors, two Guardian monitors and 180 sensors) was donated by Medtronic Austria.

\section{Author details}

'Department of Medicine III, Division of Gastroenterology and Hepatology, ICU 13H1, Medical University of Vienna, Waehringer Guertel 18-20, 1090 Vienna, Austria. ${ }^{2}$ Department of Emergency Medicine, Medical University of Vienna, Waehringer Guertel 18-20, 1090 Vienna, Austria.

\section{Authors' contributions}

RB collected data, carried out the statistical analyses and interpretation, and drafted the manuscript. GA collected data and revised the manuscript critically. $\mathrm{HH}$ performed statistical analyses. $\mathrm{CM}$ and $\mathrm{UH}$ designed and coordinated the study, collected data and helped to draft the manuscript. All authors read and approved the final manuscript.

\section{Competing interests}

The authors declare that they have no competing interests. All authors were paid by the Medical University of Vienna.

Received: 30 April 2012 Revised: 10 September 2012

Accepted: 2 October 2012 Published: 2 October 2012

\section{References}

1. van den Berghe $G$, Wouters $P$, Weekers $F$, Verwaest $C$, Bruyninckx F, Schetz M, Vlasselaers D, Ferdinande P, Lauwers P, Bouillon R: Intensive insulin therapy in critically ill patients. N Engl J Med 2001, 345:1359-1367.

2. Sacks DB: Therapy: intensive glucose control in the ICU: is sugar nice? Nat Rev Endocrinol 2009, 5:473-474.

3. Egi M, Bellomo R, Stachowski E, French CJ, Hart G: Variability of blood glucose concentration and short-term mortality in critically ill patients. Anesthesiology 2006, 105:244-252.

4. Mackenzie I, Whitehouse T, Nightingale P: The metrics of glycaemic control in critical care. Intensive Care Med 2011, 37:435-443.

5. Ali NA, O'Brien JM, Dungan K, Phillips G, Marsh CB, Lemeshow S, Connors AF, Preiser J-C: Glucose variability and mortality in patients with sepsis. Crit Care Med 2008, 36:2316-2321.

6. Dossett LA, Cao H, Mowery NT, Dortch MJ, Morris JM, May AK: Blood glucose variability is associated with mortality in the surgical intensive care unit. Am Surg 2008, 74:679-685.

7. Meyfroidt G, Keenan DM, Wang X, Wouters PJ, Veldhuis JD, Van den Berghe $G$ : Dynamic characteristics of blood glucose time series during the course of critical illness: effects of intensive insulin therapy and relative association with mortality. Crit Care Med 2010, 38:1021-1029.

8. Krinsley JS: Glycemic variability: a strong independent predictor of mortality in critically ill patients. Crit Care Med 2008, 36:3008-3013.

9. Hermanides J, Vriesendorp TM, Bosman RJ, Zandstra DF, Hoekstra JB, DeVries JH: Glucose variability is associated with intensive care unit mortality. Crit Care Med 2010, 38:838-842.

10. Meynaar IA, Eslami S, Abu-Hanna A, van der Voort P, de Lange DW, de Keizer N: Blood glucose amplitude variability as predictor for mortality in surgical and medical intensive care unit patients: a multicenter cohort study. J Crit Care 2012, 27:119-124.
11. Waeschle RM, Moerer $O$, Hilgers R, Herrmann P, Neumann P, Quintel M: The impact of the severity of sepsis on the risk of hypoglycaemia and glycaemic variability. Crit Care 2008, 12:R129.

12. Risso A, Mercuri F, Quagliaro L, Damante G, Ceriello A: Intermittent high glucose enhances apoptosis in human umbilical vein endothelial cells in culture. Am J Physiol Endocrinol Metab 2001, 281:924-930.

13. Monnier L, Mas E, Ginet C, Michel F, Villon L, Cristol J-P, Colette C Activation of oxidative stress by acute glucose fluctuations compared with sustained chronic hyperglycemia in patients with type 2 diabetes. JAMA 2006, 295:1681-1687.

14. Hirsch IB, Brownlee M: Should minimal blood glucose variability become the gold standard of glycemic control? J Diabetes Complications 2005, 19:178-181.

15. Krinsley JS: Glycemic variability and mortality in critically ill patients: the impact of diabetes. J Diabetes Sci Technol 2009, 3:1292-1301.

16. Bochicchio GV, Scalea TM: Glycemic control in the ICU. Adv Surg 2008, 42:261-275.

17. Corstjens AM, Ligtenberg JJM, van der Horst ICC, Spanjersberg R, Lind JSW, Tulleken JE, Meertens JHJM, Zijlstra JG: Accuracy and feasibility of pointof-care and continuous blood glucose analysis in critically ill ICU patients. Crit Care 2006, 10:R135.

18. Brunner R, Kitzberger R, Miehsler W, Herkner H, Madl C, Holzinger U: Accuracy and reliability of a subcutaneous continuous glucosemonitoring system in critically ill patients. Crit Care Med 2011, 39:659-664.

19. Lundelin K, Vigil L, Bua S, Gomez-Mestre I, Honrubia T, Varela M: Differences in complexity of glycemic profile in survivors and nonsurvivors in an intensive care unit: a pilot study. Crit Care Med 2010, 38:849-854.

20. Goldstein B, Fiser DH, Kelly MM, Mickelsen D, Ruttimann U, Pollack MM: Decomplexification in critical illness and injury: relationship between heart rate variability, severity of illness, and outcome. Crit Care Med 1998, 26:352-357.

21. Holzinger U, Warszawska J, Kitzberger R, Herkner H, Metnitz PGH, Madl C: Impact of shock requiring norepinephrine on the accuracy and reliability of subcutaneous continuous glucose monitoring. Intensive Care Med 2009, 35:1383-1389.

22. Holzinger U, Warszawska J, Kitzberger R, Wewalka M, Miehsler W, Herkner H, Madl C: Real time continuous glucose monitoring in critically ill patients - a prospective, randomized trial. Diabetes Care 2010, 33:467-72.

23. Holzinger U, Feldbacher M, Bachlechner A, Kitzberger R, Fuhrmann V, Madl C: Improvement of glucose control in the intensive care unit: an interdisciplinary collaboration study. Am J Crit Care 2008, 17:150-156.

24. Van den Berghe G, Wilmer A, Hermans G, Meersseman W, Wouters PJ, Milants I, Van Wijngaerden E, Bobbaers H, Bouillon R: Intensive insulin therapy in the medical ICU. N Engl J Med 2006, 354:449-461.

25. Danne T, de Valk HW, Kracht T, Walte K, Geldmacher R, Solter L, von dem Berge W, Welsh ZK, Bugler JR, Lange K, Kordonouri O: Reducing glycaemic variability in type 1 diabetes self-management with a continuous glucose monitoring system based on wired enzyme technology. Diabetologia 2009, 52:1496-1503.

26. Krinsley JS, Grover A: Severe hypoglycemia in critically ill patients: risk factors and outcomes. Crit Care Med 2007, 35:2262-2267.

27. Harmsen R, Van Braam Houckgeest F, Spronk P, Schultz M, Abu-Hanna A: Blood glucose variability, measured as mean absolute glucose, strongly depends on the frequency of blood glucose level measurements. Crit Care 2011, 15:P392.

28. Churruca J, Vigil L, Luna E, Ruiz-Galiana J, Varela M: The route to diabetes: Loss of complexity in the glycemic profile from health through the metabolic syndrome to type 2 diabetes. Diabetes Metab Syndr Obes 2008, 1:3-11.

29. Ogata H, Tokuyama K, Nagasaka S, Ando A, Kusaka I, Sato N, Goto A, Ishibashi S, Kiyono K, Struzik ZR, Yamamoto Y: Long-range negative correlation of glucose dynamics in humans and its breakdown in diabetes mellitus. Am J Physiol Regul Integr Comp Physiol 2006, 291:1638-1643.

30. Breton MD, Kovatchev BP: Impact of blood glucose self-monitoring errors on glucose variability, risk for hypoglycemia, and average glucose control in type 1 diabetes: an in silico study. J Diabetes Sci Technol 2010, 4:562-570.

31. Kalfon $\mathrm{P}$, Chilles M: Impact of the type of glucose monitoring on the assessment of glycemic variability in critical care patients. Crit Care 2012, 16:P169. 
32. Corstjens AM, Ligtenberg JJ, van der Horst IC, Spanjersberg R, Lind JS,

Tulleken JE, Meertens JH, Zijlstra JG: Accuracy and feasibility of point-ofcare and continuous blood glucose analysis in critically ill ICU patients. Crit Care 2006, 10:R135.

doi:10.1186/cc11657

Cite this article as: Brunner et al:: Glycemic variability and glucose

complexity in critically ill patients: a retrospective analysis of

continuous glucose monitoring data. Critical Care 2012 16:R175.

Submit your next manuscript to BioMed Central and take full advantage of:

- Convenient online submission

- Thorough peer review

- No space constraints or color figure charges

- Immediate publication on acceptance

- Inclusion in PubMed, CAS, Scopus and Google Scholar

- Research which is freely available for redistribution

Submit your manuscript at 